\title{
Ojales nerviosos palmares de Hartmann. Revisión y clasificación topográfica
}

\author{
H. BIANCHI \\ $3^{\text {RA }}$ CÁtedra de ANATOMÍA. FACULTAD DE MEDiCina UBA. \\ CÁTEDRA ANATOMÍA FUNDACIÓN HA BARCELÓ. \\ Correspondencia: \\ 3ra Cátedra de Anatomía \\ Facultad de Medicina UBA \\ Facultad de Medicina Fundación HA Barceló. \\ Buenos Aires. Argentina. \\ e-mail: hbianchi@fmed.uba.ar
}

\begin{abstract}
Los autores realizan un trabajo basado en la observación de las formaciones que aparecen en los nervios digitales palmares conocidas como ojales de Hartmann. Los resultados ponen de manifiesto que se trata de variaciones anatómicas habituales, con frecuencia superiores a la unidad en un mismo espécimen y que afectan tanto a las arterias digitales comunes como a las arcadas arteriales superficial y profunda. Así mismo se ha encontrado a la anastomosis superficial entre los nervios mediano y cubital tomando parte en la formación del ojal. Basados en estos hallazgos, se propone una clasificación morfológica, haciendo énfasis en la importancia que dichas formaciones adquieren en numerosas intervenciones quirúrgicas de la mano.
\end{abstract}

Palabras clave: ojales nerviosos de Hartmann, anatomía, mano.
The authors present a paper about the observations of the formations in the palmar digital nerves known as Hartmann's loops. The findings show that they are habitual anatomic variations frequently superior to the unit in a same specimen, being the digital palmar common arteries so involved as the superficial and deep palmar arches in the formation of the loop. The median ulnar superficial anastomoses were founded participating in the configuration of the loop. Based on this findings, a morphologic classification sets out, making emphasis in the importance that this formations acquire in numerous operations of the hand.

Key Words: Hartmann's loops, anatomy.

Rev. Iberam. Cir. Mano - Vol. 34 • Núm. 2 - noviembre 2006 (56-60)

\section{INTRODUCCIÓN}

L

os ojales nerviosos descritos por Hartmann (citado por Hovelacque ${ }^{1}$ ) son mencionados en la literatura como hallazgos ocasionales $^{2,3}$, excepción hecha de las observaciones realizadas por Gotani y Gilbert ${ }^{4}$ y las llevadas a cabo por el propio autor ${ }^{5}$.

En este trabajo se presenta el resultado de un estudio anatómico y se establece una clasificación de dichos ojales en base a su morfolo- gía y a las arterias alrededor de las cuales se forman, buscando facilitar la comprensión, la localización y el reconocimiento fácil de los mismos por parte del cirujano.

\section{MATERIAL Y MÉTODO}

La investigación ha sido realizada en la Tercera Unidad Docente de Anatomía del Departamento de Anatomía de la Facultad de Medicina 


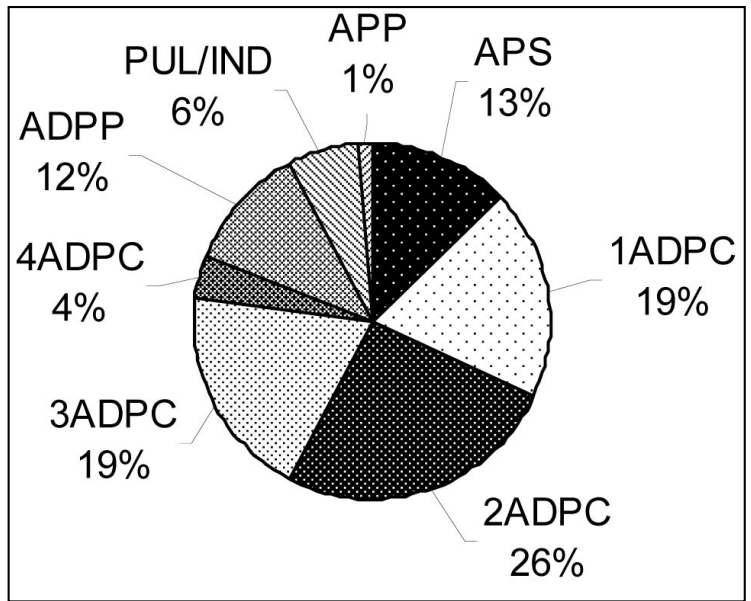

Tabla 1: 1-2-3-4 ADPC :1-2-3-4 arterias digitales palmares comunes. APS: arco palmar superficial. APP: arco palmar profundo. ADPP: arteria digital palmar propia medial del dedo pequeño. PUL/IND: tronco arterial pulgar índice

de la Universidad de Buenos Aires y la Cátedra de Anatomía de la Facultad de Medicina del Instituto de Ciencias de la Salud de la Fundación HA Barceló. En el estudio se emplearon 30 preparaciones de manos frescas y 17 formoladas, correspondientes a especimenes adultos sin distinción de sexo o edad. Los miembros frescos fueron inyectados con látex natural coloreado con pigmentos de la serie Imperan (Hoescht) y luego formolados al 15\%. Finalmente fueron aclarados con peróxido de hidrógeno al 10\%. La disección se realizó con gafas lupa de 2x.

\section{RESULTADOS}

a) En tres de las piezas disecadas no aparecieron ojales nerviosos, mientras que en 44 (93.6\%) sí los hubo.

b) El número total de ojales nerviosos disecados fue de 78. En 25 manos (56.8\%) aparecieron unidades aisladas por espécimen, mientras que en 19 manos $(43,18 \%)$ existió más de un ojal por pieza disecada.

c) En las manos con varios ojales, la distribución fue la siguiente: 8 manos tenían 2 ojales $(42.1 \%), 7$ manos 3 ojales $(36.8 \%)$ y 4 manos 4 ojales $((21,10 \%)$

d) La tabla 1 muestra la frecuencia con la que los distintos ejes arteriales de la mano fueron incluidos en un ojal, siendo la segunda

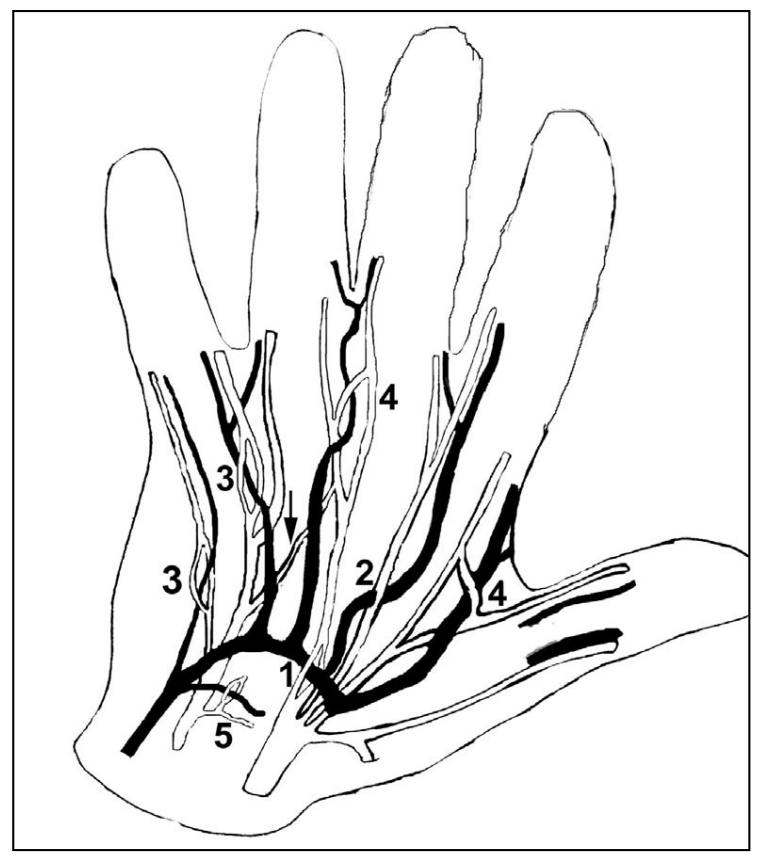

Figura 1: Esquema de la distribución de los ojales: 1: APS: arco palmar superficial. 2: ojales distales cerrados. 3: ojales distales abiertos II a. 4: ojales distales abiertos II b. 5: ojal con APP - arco palmar profundo-. Flecha: anastomosis superficial mediano cubital.

arteria digital palmar común (2ADPC) la que más veces estuvo implicada. (los porcentajes se establecen en relación con el número total de ojales encontrados).

\section{CLASIFICACIÓN}

Los ojales han sido divididos en superficiales (sensitivos) y profundos (motores). Su disposición general se muestra en la Figura 1. Los porcentajes se estimaron en relación al número total de ojales.

\section{A) Ojales superficiales}

Los ojales superficiales fueron encontrados en el arco palmar superficial (APS) y sus ramas de la siguiente manera:

A.1. Ojales proximales. Aparecieron $10 \mathrm{ca}-$ sos $(12.8 \%)$, de los que $7(70 \%)$ mostraban el ojal en un nervio sensitivo digital palmar común (NDPC) derivado del mediano (Figura 2), y $3(30 \%)$, en el NDPC derivado del cubital.

A.2. Ojales distales. Se formaron en torno a las ADPC en 67 casos (85.8\%). Por su morfología fueron subdivididos en dos grupos: 


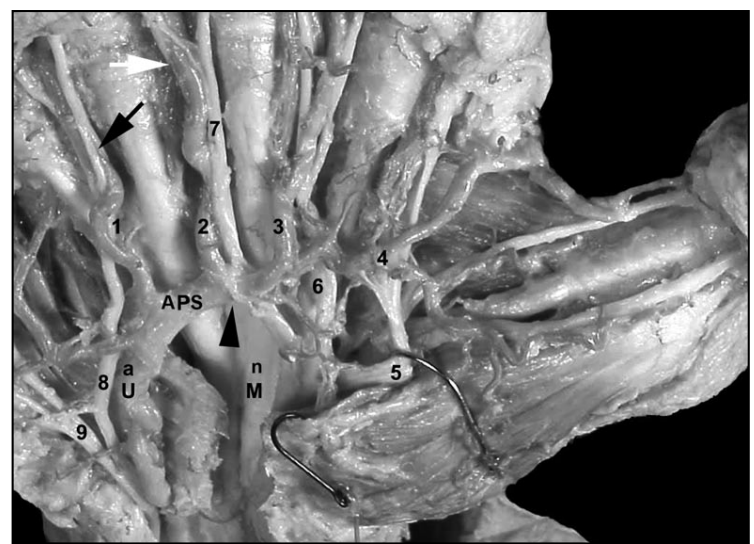

Figura 2: $n$ M: nervio mediano, a $U$ : arteria ulnar. 1 a 4 arterias digitales palmares comunes y 5 a 8 nervios digitales palmares propios.

Triángulo negro: ojal nervioso en relación con el APS. Flecha negra: ojal distal abierto II a - flecha blanca: ojal distal abierto II b.

- A.2.1. Distal I, u ojales cerrados: 10 casos $(14.9 \%)$, en los que la arteria perforaba el centro del NDPC (Figura 3).

- A.2.2. Distal II, u ojales abiertos: 57 casos $(85.1 \%)$, en los que la arteria perforaba un ojal formado a expensas del nervio sensitivo digital palmar propio (NDPP). A su vez, estos ojales distales tipo II o abiertos, se subdividieron en:

- A.2.2.1. Distales II a (35 casos -61\%), cuando la arteria atravesaba el NDPP (Figura 2).

- A.2.2.2. Distal II b (22 casos - 39\%-), cuando la arteria perforaba un ojal resultante de la anastomosis entre dos NDPP vecinos (Figura 2).

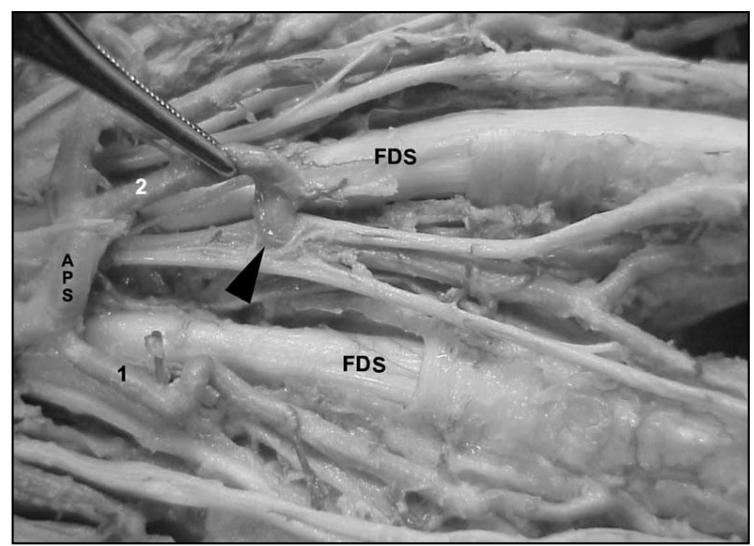

Figura 3: FDS: tendón flexor digital superficial. APS: arco palmar superficial - 1 y 2 arterias digitales palmares comunes - 3: nervio digital palmar común. Triángulo negro: ojal distal cerrado.

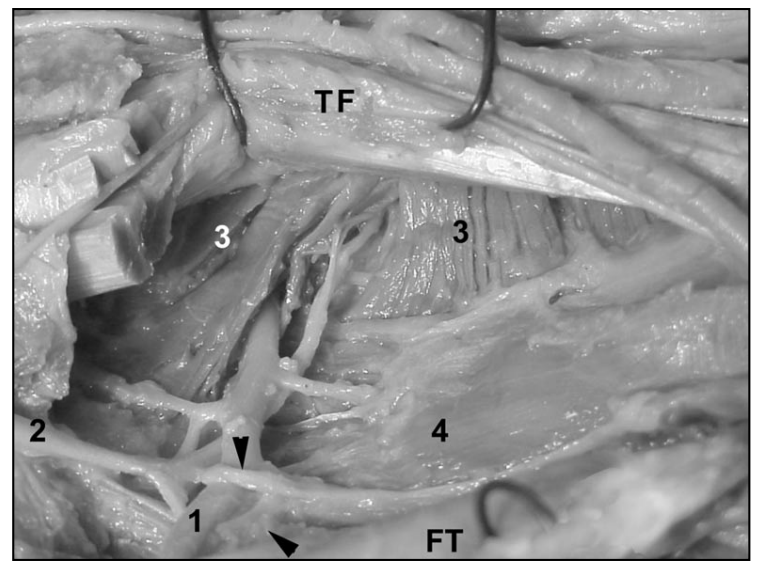

Figura 4: FT: tendones flexores palmares traccionados al cenit. 1: APP: arco palmar profundo. 2: rama profunda del nervio cubital. 3: adductor pollicis. 4: músculos interoseos palmares. Triángulos negros muestran el ojal profundo.

\section{B. Ojales profundos}

Los ojales profundos ( 1 caso, 1,28,\%), fueron hallados en el ramo motor del nervio cubital, perforado por el arco palmar profundo (APP) (Figura 4).

Participación de la anastomosis superficial mediano-cubital en la formación del ojal nervioso.

En 4 casos $(9 \%)$ se demostró la participación de la anastomosis superficial mediano-cubital en la palma de la mano. En 3 de ellos (Figura 5), el ramo se originó en el nervio mediano y en 1 en el nervio cubital. El ramo anastomótico alcanzaba la rama lateral o medial del ojal según se origine en el nervio mediano ó en el ulnaris, cerca del punto en que se dividía para dejar paso a la arteria.

\section{Longitud de los ojales nerviosos}

En 3 casos $(6,80 \%)$, los ojales fueron largos, alcanzando hasta $7 \mathrm{~cm}$. (Figura 6), mientras que el resto resultó ser corto, con longitudes de 2 a $3 \mathrm{~cm}$.

\section{DISCUSIÓN}

De los resultados encontrados en nuestro trabajo se deduce que estas formaciones anatómicas constituyen un hallazgo muy frecuente, que 


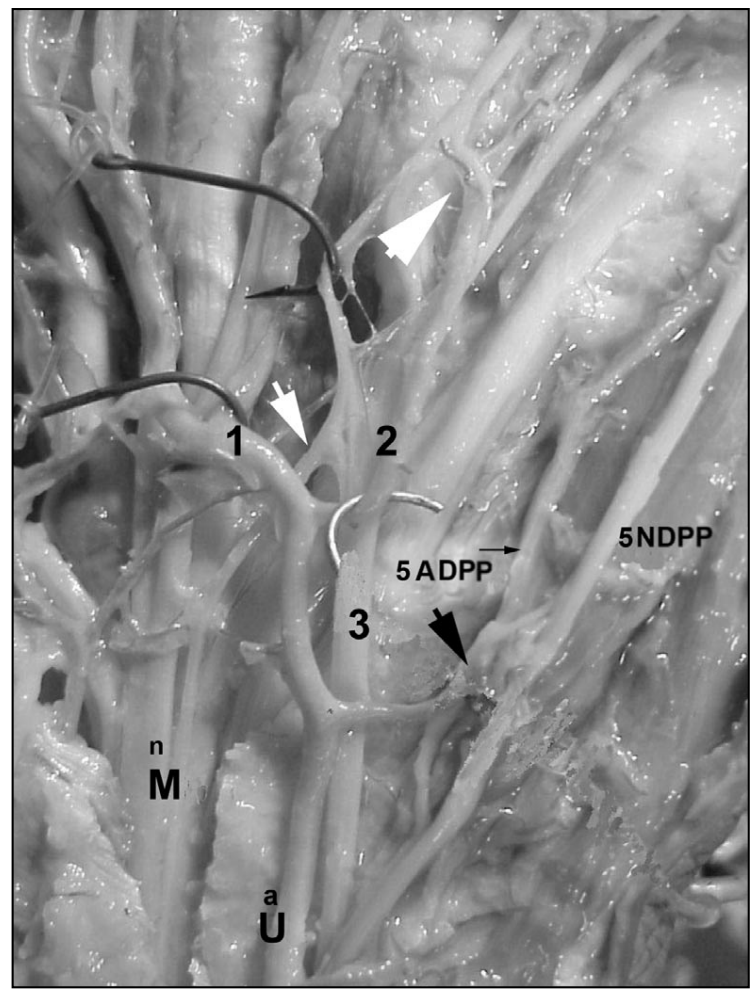

Figura. 5: $n$ M: nervio mediano - a U: arteria ulnar 1: APS: arco palmar superficial. 2: ADPC primera arteria digital palmar común. 3: nervio digital palmar común del cuarto espacio. 4 ADPP arteria digital palmar propia medial del dedo pequeño (flecha pequeña) 5 NDPP: nervio digital palmar propio medial del dedo pequeño. La flecha blanca pequeña muestra el ramo anastomótico superficial mediano ulnar; la grande muestra el ojal nervioso. La flecha negra muestra un ojal nervioso del NDPP medial del dedo pequeño en relación con la ADPP medial del dedo pequeño.

cuantitativamente son importantes y que a menudo aparecen de manera múltiple en una misma mano. Coincidimos en este sentido con las observaciones de Hovelacque ${ }^{1}$.

Los nervios implicados en la formación de los ojales son los DPC y el DPP. Las arterias que perforaban estos ojales fueron el APS y el APP, las ADPC, la ADPP medial del quinto dedo, la arteria radial del índice y el tronco común pulgar-índice. No hemos encontrado ojales de Hartmann relacionados con las ADPP del pulgar, como tampoco han sido encontrados en otros estudios realizados por Gotani y Gilbert ${ }^{4}$.

La participación de la anastomosis superficial mediano cubital en la formación del ojal nervioso, es un hecho que con excepción de Leiro $^{6}$, no había sido mencionado en trabajos

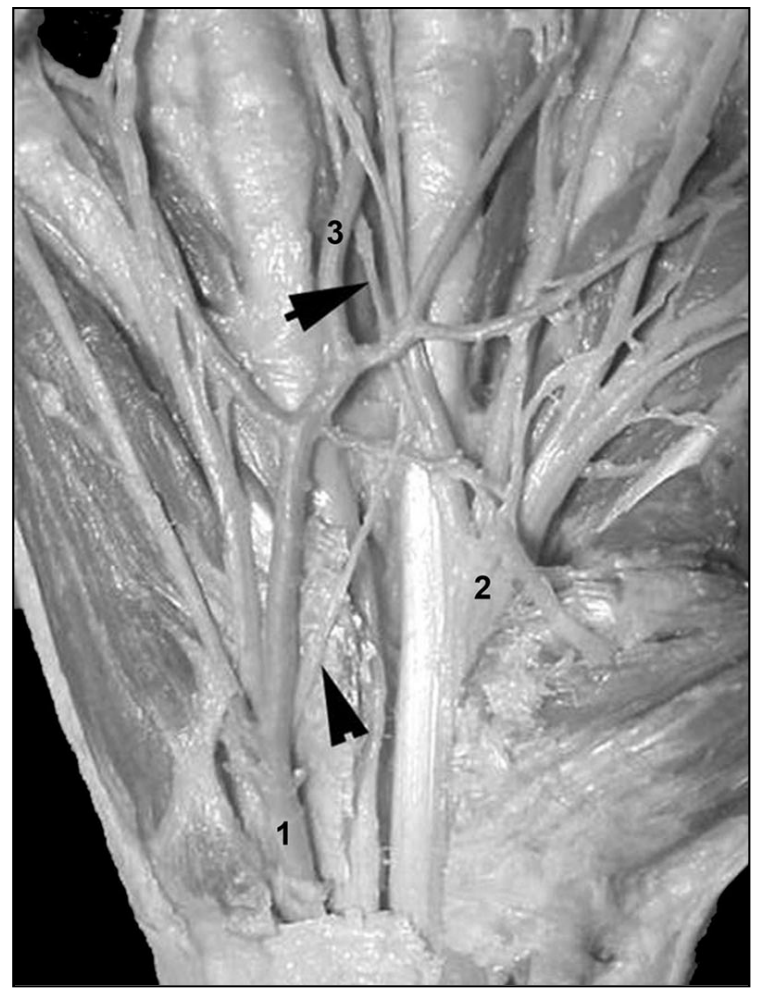

Fig. 6: 1: arteria ulnar- 2: nervio mediano - 3: segunda arteria digital palmar común. Flecha triangular: anastomosis superficial mediano ulnar participando en la formación del ojal nervioso largo, señalado por la flecha negra.

previos. Tampoco hemos encontrado en la literatura consultada la existencia de ojales profundos en ejes nerviosos motrices.

En lo que concierne a la longitud, los ojales largos no son frecuentes. Sin embargo, su configuración puede conducir a engaño al ser identificados indebidamente durante la disección como un nervio DPP.

La clasificación que presentamos basada en la morfología y localización de los ojales y su relación con las arterias, creemos que es fácil de recordar, de comprender y de aplicar.

En fin, desde una perspectiva quirúrgica, los ojales de Hartmann deberían ser considerados como obstáculos potenciales en el desarrollo de numerosas intervenciones quirúrgicas en la mano, especialmente las que implican disección vasculo-nerviosa. Éste es el caso de la disección de colgajos palmares, como el de Littler, en los que el pedículo ha de ser liberado para ser posteriormente rotado.

De la misma forma, estas estructuras habrán de ser recordadas en la disección de procesos 
retráctiles como la enfermedad de Dupuytren, en los que a menudo están incluidos los paquetes vasculo-nerviosos digito-palmares en las bandeletas pretendinosas o en las bridas digita- les retraídas. En estos casos, el riesgo de isquemia digital y de alteración sensitiva en las zonas tributarias se vería incrementado por la presencia de dichos ojales.

\section{BIBLIOGRAFÍA}

1. Hovelacque A: Des Nerfs Crâniens et Rachidiens et du Système Grand Sympathique, T2, ed Gaston Doin et Cia, Libraire Octave Dion, Paris, 1927: 455

2. Sener M Yildiz M Aydin H: Anomalous digital nerve loop: a case report, Microsurgery 1998; 18: $170-171$
3. Spinner RJ Varela Ch Urbaniak D: Digital Nerve Penetration by digital artery in a patient with neurovascular symptoms a case report, J Hand Surgery 1996; 21 A:1101-1103

4. Gotani H-Gilbert A: Hartmann's Boutonnière: an anatomical study of digital neural loop pe- netrations in the palm, J Hand Surg 1999: 24B: 35-37

5. Bianchi $\mathrm{H}$ : Asas nerviosas de los nervios digitales palmares, Rev Chil Anat, 2002; 20: 95.

6. Leiro R Torres MP Séller A Ojales nerviosos para las arterias digitales palmares, Púb. Soc. Rioplatense Anatomía, 1983, T XII, 86. 